sive der allenfalls notwendigen Entsorgung einzelner Materialkomponenten (z. B. Konzentrate, Schlämme etc.).

In unserem Modellfall Schweizerhalle wird mit einer Kombination verschiedener Verfahrenstechniken gearbeitet:

Das gesamte zu reinigende Bodenmaterial wird in einer eigens für das Projekt SABO konzipierten Flotationsanlage behandelt. Bei der Flotation werden in mehreren Stufen mit physikalisch-chemischen Verfahren die Schadstoffe mit den Feinstanteilen (Schluff) vom gröberen Bodenmaterial getrennt, was einer Aufkonzentration der Schadstoffe gleichkommt. Die Reinigung des Prozessabwassers sowie die Abluftreinigung müssen als flankierende Massnahmen in den Bodenreinigungsprozess integriert werden. Der erzielbare Reinigungsgrad mit diesem speziell umweltschonenden Verfahren liegt je nach Schadstoffart und -konzentration zwischen $80 \%$ und $99 \%$. Bei sehr hohem Verschmutzungsgrad ist eine Nachbehandlung jedoch unumgänglich. Als geeignete Methoden erweisen sich hierbei chemische oder zumindest teilweise mikrobiologische Nachreinigungsverfahren.

Für die Entsorgung der Schlämme mit den aufkonzentrierten Schadstoffen gelangen materialspezifische Verfahren zur An- wendung. Anhand des Projektes SABO zeigt sich, dass eine thermische Behandlung verfahrenstechnisch und ökologisch gesehen häufig die einzig gangbare Möglichkeit darstellt.

Wo stehen wir im Sanierungsprojekt Schweizerhalle heute? Die in einer eigens dafür konzipierten Halle - zur Vermeidung von Geruchsbelästigungen wird im Innern mit einem kleinen Unterdruck gearbeitet ist die Flotationsanlage fertiggestellt worden. Die Pilotphase zur Optimierung der verfahrenstechnischen Abläufe wird in 1-2 Monaten abgeschlossen; mit der eigentlichen Bodenreinigung kann somit in diesem Frühjahr, rund $31 / 2$ Jahre nach dem Brand, begonnen werden. Der Zeitbedarf für diese letzte Projektphase wird mit nochmals rund 2 Jahren veranschlagt. Für die gesamten verfahrenstechnischen Abklärungen zur Reinigung des Bodens, begonnen im Reagenzglas im Oktober 1987, wurden also $c a .21 / 2$ Jahre investiert.

Wie hoch kommen jedoch die Kosten für eine komplette Bodenreinigungsanlage zu stehen? Der Investitionsbedarf für eine Flotationsanlage inklusive betonierter Wanne, Halle sowie Ver- und Entsorgungsleitungen kann mit rund $10 \mathrm{Mio} \mathrm{sFr}$. veranschlagt werden. Mit der notwendigen Infrastruktur (Zwischenlagerplätze, La- bors für Analytik, Sicherheits- und Qualitätskontrollsystem, Nachreinigungsanlagen etc.) ist mit einem Gesamtaufwand von 15-20 Mio.sFr. zu rechnen. Für eine einzelne, kleinere Bodenverschmutzung wäre dieser Aufwand deshalb unverhältnismässig. Der Bau von zentral gelegenen, regionalen Bodenentsorgungszentren käme daher billiger zu stehen und die Qualität der Arbeiten könnten besser überwacht werden als bei irgendwelchen improvisierten Lösungen.

Mit der beschriebenen Flotationsanlage wird zur Zeit ein erstes Bodenentsorgungszentrum in der Schweiz in Betrieb genommen. Allein für Sanierung der beim Brand des Chemielagers in Schweizerhalle entstandenen Bodenverschmutzung ist die Anlage sicher für die nächsten zwei Jahre voll ausgelastet. Zudem muss der Standort - die Anlage steht mitten in einem chemischen Industriebetrieb - zumindest mittelfristig überprüft werden.

Wie auch in anderen Sparten der Umwelttechnik fehlen uns also immer noch die elementarsten Teile der Infrastruktur zur Behandlung von Umweltschäden und zur Minimierung der Belastung unserer Umwelt. Ein Umdenken und Mut zum Handeln sind daher angezeigt. Die Zeit drängt.

\title{
Abfallminderung, ein zentrales Anliegen der chemischen Industrie
}

\author{
Urs Gujer*
}

\section{Abfälle aus der chemischen Industrie}

\subsection{Abfallquellen}

Die chemische Industrie unterscheidet sich in einem Punkt wesentlich von anderen, ebenfalls hochtechnischen Industriezweigen: durch die Eigenheiten chemischer Umsetzungen. Bei diesen entstehen durch chemische Reaktionen neue Stoffe. Was für neue Stoffe, mit Bezug auf die Ausgangsstoffe, dabei entstehen, hängt von den Energieverhältnissen ab.

Naturgesetze bedingen, dass es auch in der Chemie treibende Kräfte gibt, durch

\footnotetext{
Korrespondenz: Dr. U. Gujer

Ciba-Geigy AG

CH-4002 Basel
}

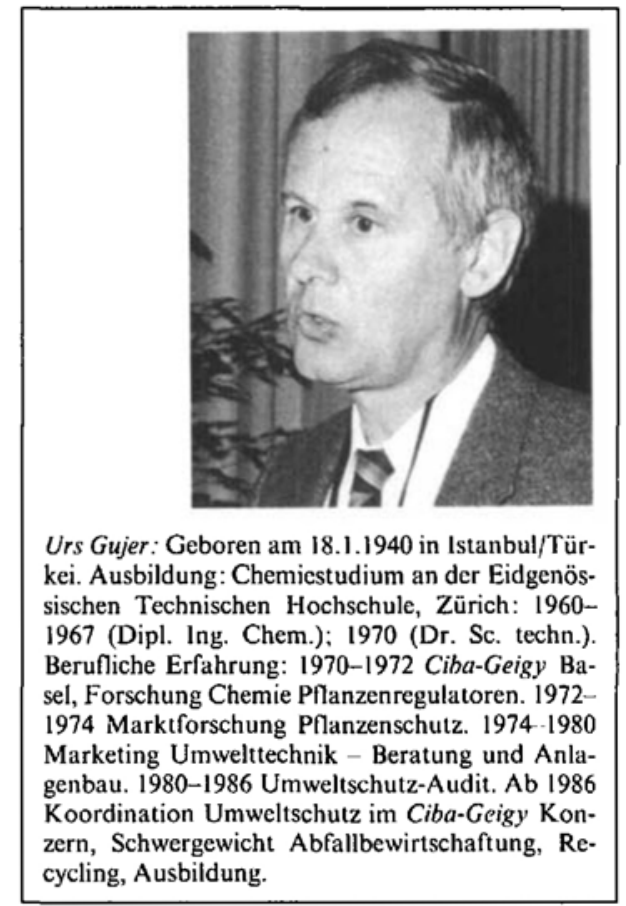

Hilfsstoffe müssen ebenfalls nach Abschluss der Reaktion vom gewünschten Produkt abgetrennt werden und sind typische, chemiespezifische Abfälle.

Es ist eine oft übersehene Tatsache, dass sich Materie nicht vernichten lässt. Sie 
sive der allenfalls notwendigen Entsorgung einzelner Materialkomponenten (z. B. Konzentrate, Schlämme etc.).

In unserem Modellfall Schweizerhalle wird mit einer Kombination verschiedener Verfahrenstechniken gearbeitet:

Das gesamte zu reinigende Bodenmaterial wird in einer eigens für das Projekt SABO konzipierten Flotationsanlage behandelt. Bei der Flotation werden in mehreren Stufen mit physikalisch-chemischen Verfahren die Schadstoffe mit den Feinstanteilen (Schluff) vom gröberen Bodenmaterial getrennt, was einer Aufkonzentration der Schadstoffe gleichkommt. Die Reinigung des Prozessabwassers sowie die Abluftreinigung müssen als flankierende Massnahmen in den Bodenreinigungsprozess integriert werden. Der erzielbare Reinigungsgrad mit diesem speziell umweltschonenden Verfahren liegt je nach Schadstoffart und -konzentration zwischen $80 \%$ und $99 \%$. Bei sehr hohem Verschmutzungsgrad ist eine Nachbehandlung jedoch unumgänglich. Als geeignete Methoden erweisen sich hierbei chemische oder zumindest teilweise mikrobiologische Nachreinigungsverfahren.

Für die Entsorgung der Schlämme mit den aufkonzentrierten Schadstoffen gelangen materialspezifische Verfahren zur An- wendung. Anhand des Projektes SABO zeigt sich, dass eine thermische Behandlung verfahrenstechnisch und ökologisch gesehen häufig die einzig gangbare Möglichkeit darstellt.

Wo stehen wir im Sanierungsprojekt Schweizerhalle heute? Die in einer eigens dafür konzipierten Halle - zur Vermeidung von Geruchsbelästigungen wird im Innern mit einem kleinen Unterdruck gearbeitet ist die Flotationsanlage fertiggestellt worden. Die Pilotphase zur Optimierung der verfahrenstechnischen Abläufe wird in 1-2 Monaten abgeschlossen; mit der eigentlichen Bodenreinigung kann somit in diesem Frühjahr, rund $31 / 2$ Jahre nach dem Brand, begonnen werden. Der Zeitbedarf für diese letzte Projektphase wird mit nochmals rund 2 Jahren veranschlagt. Für die gesamten verfahrenstechnischen Abklärungen zur Reinigung des Bodens, begonnen im Reagenzglas im Oktober 1987, wurden also $c a .21 / 2$ Jahre investiert.

Wie hoch kommen jedoch die Kosten für eine komplette Bodenreinigungsanlage zu stehen? Der Investitionsbedarf für eine Flotationsanlage inklusive betonierter Wanne, Halle sowie Ver- und Entsorgungsleitungen kann mit rund $10 \mathrm{Mio} \mathrm{sFr}$. veranschlagt werden. Mit der notwendigen Infrastruktur (Zwischenlagerplätze, La- bors für Analytik, Sicherheits- und Qualitätskontrollsystem, Nachreinigungsanlagen etc.) ist mit einem Gesamtaufwand von 15-20 Mio.sFr. zu rechnen. Für eine einzelne, kleinere Bodenverschmutzung wäre dieser Aufwand deshalb unverhältnismässig. Der Bau von zentral gelegenen, regionalen Bodenentsorgungszentren käme daher billiger zu stehen und die Qualität der Arbeiten könnten besser überwacht werden als bei irgendwelchen improvisierten Lösungen.

Mit der beschriebenen Flotationsanlage wird zur Zeit ein erstes Bodenentsorgungszentrum in der Schweiz in Betrieb genommen. Allein für Sanierung der beim Brand des Chemielagers in Schweizerhalle entstandenen Bodenverschmutzung ist die Anlage sicher für die nächsten zwei Jahre voll ausgelastet. Zudem muss der Standort - die Anlage steht mitten in einem chemischen Industriebetrieb - zumindest mittelfristig überprüft werden.

Wie auch in anderen Sparten der Umwelttechnik fehlen uns also immer noch die elementarsten Teile der Infrastruktur zur Behandlung von Umweltschäden und zur Minimierung der Belastung unserer Umwelt. Ein Umdenken und Mut zum Handeln sind daher angezeigt. Die Zeit drängt.

\title{
Abfallminderung, ein zentrales Anliegen der chemischen Industrie
}

\author{
Urs Gujer*
}

\section{Abfälle aus der chemischen Industrie}

\subsection{Abfallquellen}

Die chemische Industrie unterscheidet sich in einem Punkt wesentlich von anderen, ebenfalls hochtechnischen Industriezweigen: durch die Eigenheiten chemischer Umsetzungen. Bei diesen entstehen durch chemische Reaktionen neue Stoffe. Was für neue Stoffe, mit Bezug auf die Ausgangsstoffe, dabei entstehen, hängt von den Energieverhältnissen ab.

Naturgesetze bedingen, dass es auch in der Chemie treibende Kräfte gibt, durch

\footnotetext{
Korrespondenz: Dr. U. Gujer

Ciba-Geigy AG

CH-4002 Basel
}

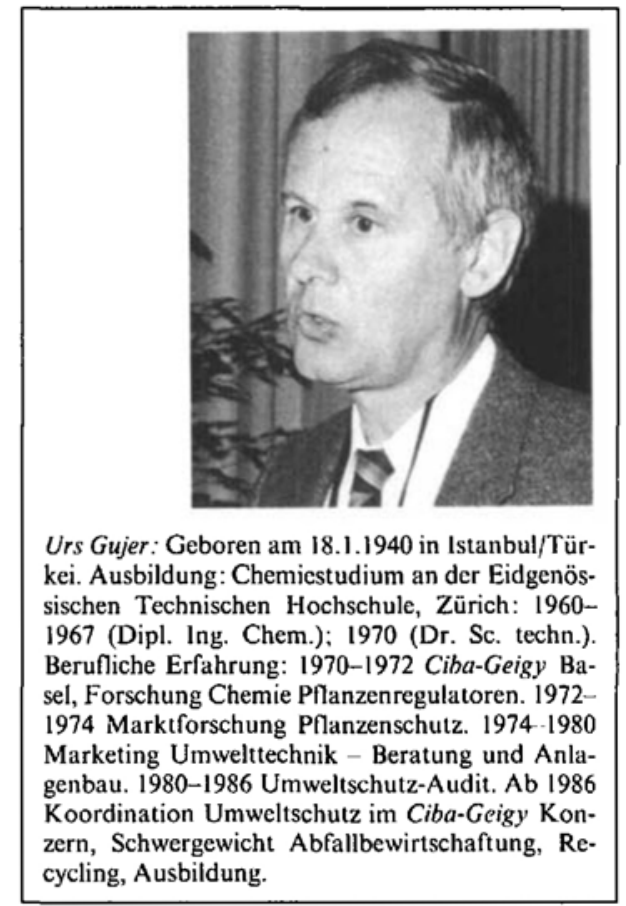

Hilfsstoffe müssen ebenfalls nach Abschluss der Reaktion vom gewünschten Produkt abgetrennt werden und sind typische, chemiespezifische Abfälle.

Es ist eine oft übersehene Tatsache, dass sich Materie nicht vernichten lässt. Sie 
wird stets nur umgewandelt und von einer Phase in eine andere transferiert. So fallen aus dem Betrieb von Anlagen zum Schutz der Umwelt, wie Abluft-oder Abwasserbehandlungsanlagen, Schlämme und Stäube an und aus Verbrennungsanlagen ergeben sich Aschen und Schlacken. Dies sind weitere produktionsspezifische Abfälle.

\subsection{Mengen}

Für die Spezialitäten-Chemie in der Schweiz typische Mengenangaben für Abfälle (bezogen auf die Menge hergestellter Produkte) sind im folgenden aufgeführt:

Abfälle aus chemischen

Umsetzungen

(inkl. Schlämme

und Stäube aus der Reinigung

von Abluft und Abwasser)

Abfall-Lösungsmittel

Metall-Schlämme

Aschen und Schlacken

Industriekehricht

$0,5-5 \%$

$2,0-15 \%$

$0-15 \%$

$1,0-5 \%$

$1,0-8 \%$

Die aufgeführten Grössenordnungen verdeutlichen, dass je nach Art eines Betriebes und der herzustellenden Produkte die Abfallmengen stark schwanken. So entstehen aus kontinuierlich geführten Prozessen, die nur einige wenige Reaktionsstufen beanspruchen, geringere Mengen an spezifischen Abfällen, als bei absatzweisen Herstellungsverfahren, welche bis zu 30 und mehr Reaktionsstufen verlangen (z.B. bei Wirkstoffen der pharmazeutischen Industrie), oder bei Prozessen, die in Mehrzweckanlagen durchgeführt werden müssen. Es ist weiter zu beachten, dass je nachdem für welchen Zweck Zahlen benötigt werden, deren Erhebung nach unterschiedlichen Gesichtspunkten erfolgt.

Die Auslegung neuer Herstellungsverfahren, die Planung von Produktions- oder Infrastrukturanlagen, Auflagen für den Schutz der Umweltkompartimente Boden, Wasser und Luft, die Planung von regionalen oder nationalen Entsorgungskapazitäten oder für die Überwachung von Stoffbewegungen erfordern unterschiedliche Arten von Daten.

Bevor die von vielen Kreisen geforderten verlässlichen Umweltbilanzen erstellt werden können - und dazu gehören Angaben über die Minderung von Abfällen -, müssen sinnvolle Definitionen gefunden und Bilanzgebiete festgelegt werden, die von allen Betroffenen akzeptiert werden. In diesem Zusammenhang sei auf die unterschiedliche Behandlung des Begriffes 'Reststoffe' in der schweizerischen [1] und deutschen [2] Gesetzgebung hingewiesen.

\section{Abfallbewirtschaftung und Abfallminderung}

Erst um 1960 erkannte man die Grenzen der Belastbarkeit der Umwelt und ergriff erste gezielte Massnahmen zu deren Schutz. Das Schwergewicht dieser Massnahmen konzentrierte sich auf nachgeschaltete Anlagen für die Behandlung von Abluft und Abwasser und der Suche nach neuen Deponien. Dies galt für die Industrie im allgemeinen, für Kommunen und im besonderen auch für die chemische Industrie. Während dieser 'additive' Umweltschutz (End-of-pipe-Umweltschutz) relativ schnell zu einer Verminderung der Belastung in den Umweltkompartimenten Luft und Wasser geführt hat, widerspiegeln die Folgen schlecht geplanter und betriebener Abfallentsorgungsanlagen die Unzulänglichkeit, mit welcher mancherorts Abfälle gehandhabt wurden. Seit Mitte der 70er Jahre ist man sich der Möglichkeiten, aber auch der Grenzen additiver Schutzmassnahmen immer besser bewusst.

Bezogen auf die Abfälle, die zwangsläufig bei der Versorgung unserer Gesellschaft mit Gütern und Dienstleistungen entstehen, gibt es nur einen vertretbaren Weg, aus dem in den westlichen Industrieländern bewusst wahrgenommenen - aber auf der ganzen Welt vorhandenen - gegenwärtigen Entsorgungsnotstand. Dieser führt über die spezifische und gesamthafte Minderung der Abfallmengen sowie einer Umwandlung der noch verbleibenden Abfälle in eine Form, bei deren endgültiger Entsorgung keine weitere Beeinträchtigung der Umwelt zu befürchten ist.

Für eine Abfallminderung sprechen unter anderem folgende Überlegungen:

- Ein grosses Abfallaufkommen ist gleichzusetzen mit der Vergeudung von meistens nicht erneuerbaren Ressourcen.

- Der Bedarf an Einsatz von Energie (ebenfalls als Ressource) für die Umwandlung und Aufarbeitung von Abfällen ist in direktem, bei verdünnten $\mathrm{Ab}$ fällen in exponentiellem Verhältnis zur behandelten Abfallmenge.

- Zunehmende Gesamtkosten für die Sammlung, Zwischenlagerung, Transport, Behandlung und Endlagerung (einschliesslich der Analytikkosten) machen eine Abfallminderung ökonomisch interessant.

- Kurz- und mittelfristig fehlen ausreichende technisch und ökologisch vertretbare Behandlungs- und Entsorgungskapazitäten

- Die zunehmenden Kosten und die Haftungspflicht für die Sanierung von Altlasten erfordern eine wirksame Vermeidungsstrategie.

In der Praxis der chemischen Industrie werden zwei Wege für eine wirksame Minderung der Abfallvolumen beschritten:

- Die Abfallbewirtschaftung als administrative und buchhalterische Massnahme und

- die Abfallminderung als Resultat einer innovativen integrierten Verfahrensentwicklung.

\subsection{Die Abfallbewirtschaftung}

Innerhalb eines Unternehmens schafft die systematische Bewirtschaftung Transparenz in das Geschehen um die Erfassung und Entsorgung von Abfällen.

Im wesentlichen umfasst die Abfallbewirtschaftung:

- Erfassen der Abfälle an der Quelle nach Qualität und Mengen,

- Abklären der Recyclingmöglichkeiten (Verwendung, Verwertung) innerhalb oder ausserhalb des Unternehmens,

- Behandlung, d.h. Unschädlichmachen der nicht verwertbaren Abfälle mit Hilfe von physikalischen, chemischen, biologischen und thermischen Verfahren,

- sichere und umweltgerechte Entsorgung dieser Abfälle (gemäss schweizerischer Gesetzgebung: Reststoffe [1]),

- Überwachen aller Schritte mittels eines Buchführungssystems, welches zu jedem Zeitpunkt Auskunft über den Verbleib der Abfälle gibt [3] [4].

Die strikte Einhaltung dieser wenigen Punkte gewährleistet eine Auskunft über Art, Menge und Verbleib der Abfälle. Sie ermöglicht eine klare Zuteilung von $\mathrm{Zu}$ ständigkeiten und Verantwortungen sowie eine Zuordnung der Kosten für die Behandlung und Entsorgung nach dem Verursacherprinzip. Obwohl eine sorgfältig betriebene Abfallbewirtschaftung auch die Frage des Recycling berücksichtigt, begrenzt sich deren Wirksamkeit auf diejenigen Rückstände von wirtschaftlich interessantem Volumen und Qualität. Hierfür sind heute schon meistens eingespielte Recyclingwege vorhanden.

Die hier beschriebene Abfallbewirtschaftung beschränkt sich im wesentlichen auf den additiven Umweltschutz. Sie schafft die Möglichkeit, spezifische Mengen- und Einzelstofffluss-Bilanzen zu erstellen, welche ihrerseits die Grundlage einer ganzheitlichen Betrachtung mit dem Ziel der Abfallvermeidung und -minderung bilden.

\subsection{Die Abfallminderung}

Aus der Überzeugung, dass eine Verbesserung der heutigen Versorgungs- und Entsorgungssituation unserer Gesellschaft einhergehen muss mit einem Wertewandel hin zu einer umweltverträglichen, qualitativen Entwicklung, stellt sich für die chemische Industrie, als Teil der Gesellschaft, die Verpflichtung, die Entwicklung ihrer Spezialitäten so zu gestalten, dass sie eine Ressourcen schonende, allen Aspekten der Sicherheit, der Arbeitshygiene und des Umweltschutzes gerechte Herstellung gewährleistet.

\subsubsection{Die integrierte \\ Verfahrensentwicklung}

Mit der Erkenntnis der Grenzen des additiven Umweltschutzes wuchs innerhalb der chemischen Industrie die Überzeu- 


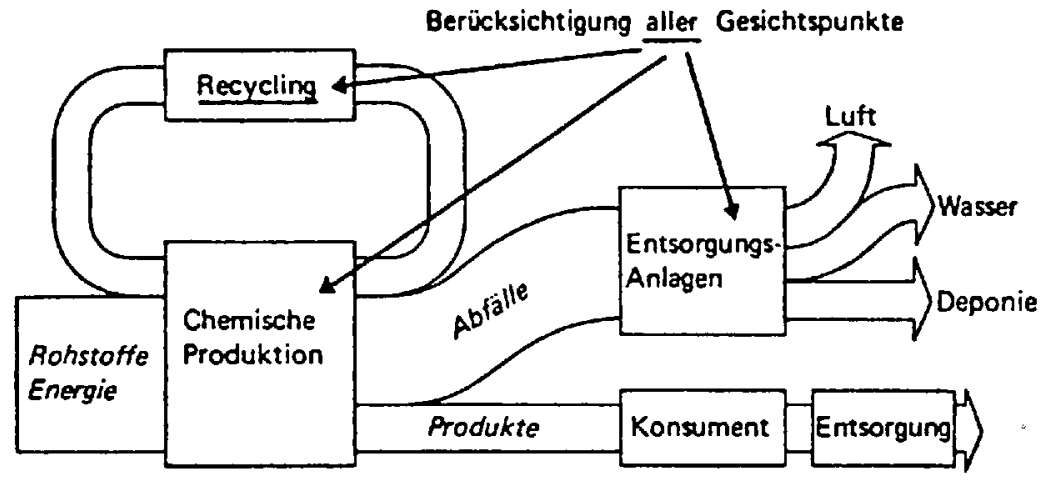

Zu optimierende Randbedingungen

- Qualitāt

- Kosten

- Unweltkonformitāt

- Energieaspekte

Fig. 1. Chemische Industrie: Integrierte Prozessentwicklung gung, dass mit der konventionellen Verfahrensbearbeitung, bei der Ausbeute, Qualität und Produktionskostenoptimierung im Vordergrund standen, jedoch das Recycling sowie die Behandlung der aus dem Produktionsprozess anfallenden Abfallströme getrennt untersucht wurden, die produktionsspezifischen Umweltprobleme nur ungenügend gelöst werden konnten.

1978 wurde daher von Ciba-Geigy die liert [5]. Das besondere Merkmal dieses Vorgehens ist, dass schon bei der Entwicklung neuer Verfahren der Produktionsprozess, angefangen mit der Art und Qualität der eingesetzten Rohstoffe, Ausschöpfung der Recyclingmöglichkeiten bis hin zu den Produktionsabfällen und deren Elimination, als Ganzes anzusehen ist (Fig. 1). Konkret heisst dies, dass für jeden Prozess die chemischen und physikalischen Reaktionsbedingungen durch detaillierte Versuche im Labor untersucht und anschliessend im Pilotmaßstab bestätigt werden. Diese dienen dazu, die Laborergebnisse im Gesamtzusammenhang zu überprüfen und schwer voraussagbare potentielle Probleme im Hinblick auf die Betriebseinführung zu erkennen. Dazu sind sorgfältige Mengenbilanzen zu erstellen, Entsorgungstechnologien gleichzeitig mit dem wickeln und neu die prozeßspezifischen Entsorgungskosten in die Produktionskostenkalkulation miteinzubeziehen. Die Suche des besten Verfahrens, welches bei minimalem Abfallausstoss und ausgewogenem Ressourcenverzehr zu einem spezifischen Produkt führt, stellt hohe Anforderungen an den Verfahrens-Chemiker.

Die Forschung nach neuen Produkten ist durch die Entwicklung neuer Herstellungsverfahren zu ergänzen. Zudem machen es die Fortschritte der Verfahrensmethodik und der apparativen Technik heute möglich und nötig, auch seit Jahren in der Produktion eingeführte Verfahren immer wieder zu überprüfen und zu verbessern.

Der Entscheidungsbaum in Fig. 2 verdeutlicht den engen Zusammenhang zwiintegrierte Verfahrensentwicklung postuoptimalen Herstellungsverfahren zu ent- schen einer chemischen Reaktion, Abfallströmen und der Produktionsanlage, mit besonderer Berücksichtigung der ProzessSicherheit [6]. Die integrierte Verfahrens- entwicklung erlaubt die dynamische Suche nach optimalen Verfahrensbedingungen durch iterative Anwendung der bekannten Werkzeuge des Verfahrens-Chemikers Weg einer neuen Chemie offen.

Es gibt keine Patentrezepte, um die bekannten Abfallprobleme zu lösen. Jeder Fall muss individuell untersucht werden und bedarf einer massgeschneiderten Lösung, die zusätzlich wegen unterschiedlicher technischer Randbedingungen für jede Produktionsanlage eigens angepasst werden muss.

\subsubsection{Der produktionsintegrierte Umweltschutz}

Ausgehend von einer ähnlich formulierten Zielsetzung der Abfallminderung hat eine Arbeitsgruppe aus Vertretern der Schweizerischen Akademie der Technischen Wissenschaften, der DECHEMA und der GVC, die sich im sogenannten 'Freiburger Kreis' zusammengeschlossen oder Ingenieurs. Bei Versagen dieser Vorgehensweise bleibt noch der schwierige

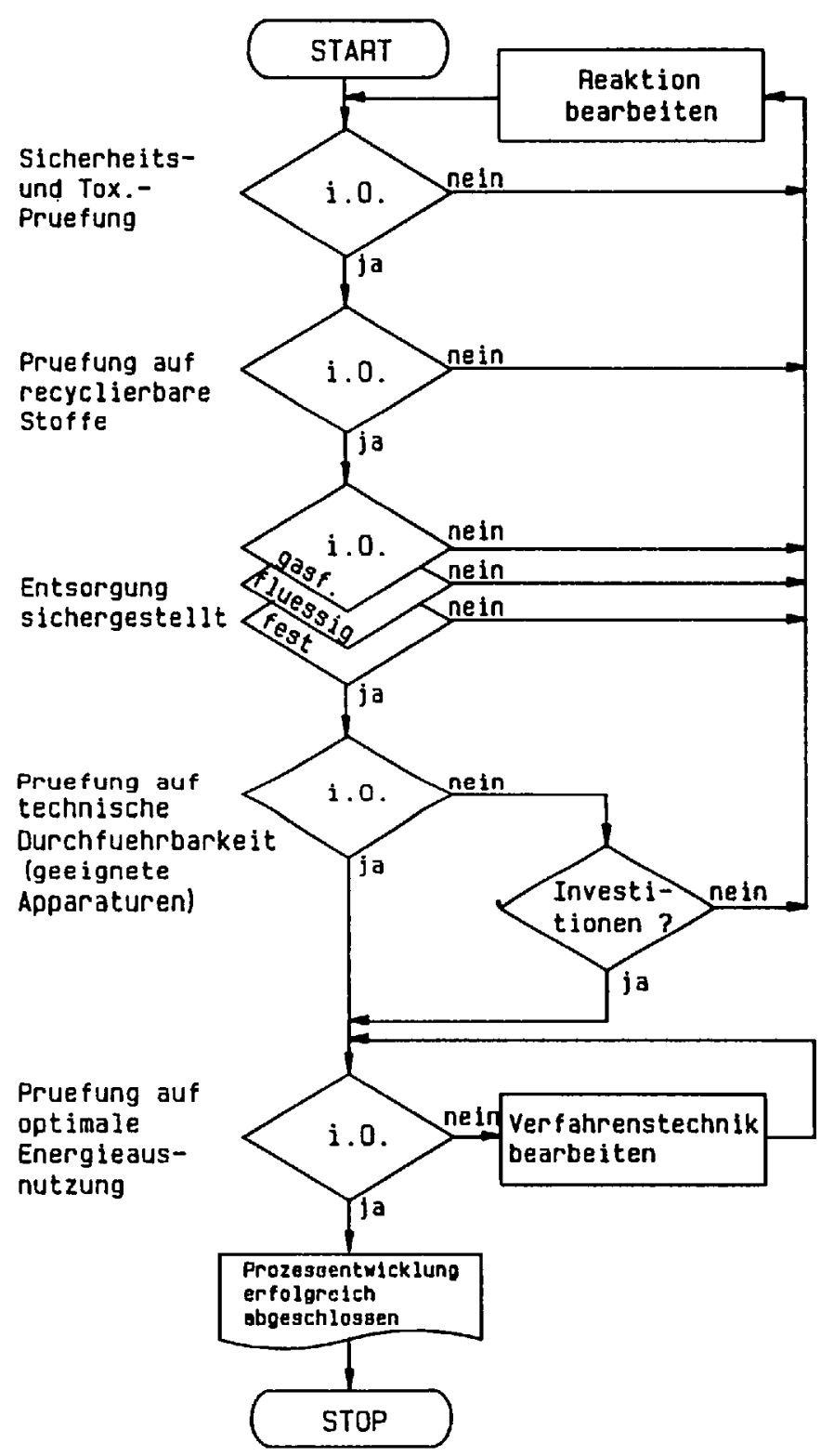

Fig. 2. Integrierte Prozessentwicklung 
hat [7], den Rahmen der Umweltschutzbemühungen bei der chemischen Produktion erneut untersucht und den Gedanken der integrierten Verfahrensentwicklung neu definiert und ausgeweitet. Unter dem neugewählten Begriff 'Produktionsintegrierter Umweltschutz' wird vom Freiburger Kreis ein Vorgehen verstanden, welches im Gegensatz zum additiven Umweltschutz den Einsatz aller chemischen, physikalischen, biologischen und technischen Möglichkeiten zur Verbesserung chemischer Prozesse vorsieht. Im Gegensatz zur integrierten Verfahrensentwicklung, welche zunächst das einzelne Herstellungsverfahren anvisiert, reicht neu der Umfang der Prozessverbesserungen von der Optimierung einzelner Prozesskomponenten und Reaktionsschritte bis hin zur Änderung ganzer Prozesse oder Prozessverbunde. Diese Verbesserungen müssen sich in der Umweltbilanz eines ganzen Werkes niederschlagen. Allerdings sind produktionstechnische Verbesserungen nur in dem Masse möglich, wie die dazu erforderlichen technischen Voraussetzungen geschaffen, und im Grossmaßstab realisiert werden können.

Für die praktische Anwendung können die integrierte Verfahrensentwicklung und der produktionsintegrierte Umweltschutz als gleichwertig und sich ergänzend angesehen werden. Ein Unterschied besteht allerdings in der Verwendung des Begriffes 'Reststoffe', für die der Freiburger Kreis, in Erweiterung der Definition im deutschen Bundes-Immissionsschutz-Gesetz, diejenigen vom Betreiber chemischer Anlagen nicht erwünschten Stoffe versteht, die in chemischen Reaktionen entstehen oder verbleiben und die zu einem Teil weiterverwendet oder verwertet werden können. Erst der nicht verwertbare Anteil wird als Abfall definiert.

\subsubsection{Das Recycling}

Bei den bisher betrachteten Möglichkeiten der Abfallminderung kommt dem Recycling eine zentrale Bedeutung zu. Recycling kann als 'operatives Element' der Ressourcenschonung bezeichnet werden. Im Rahmen der Abfallminderung werden unter dem Sammelbegriff Recycling alle Massnahmen verstanden, die zur stofflichen Wiederverwendung oder Verwertung dienen, sei es im Prozess selber, im Prozessverbund oder bei Dritten. Bei der Verwertung wird der Abfallstoff in ein Produkt mit neuen Einsatzmöglichkeiten umgewandelt.

$\mathrm{Ob}$ in jedem Fall eine Stoffrückführung oder Verwertung möglich und sinnvoll ist, hängt von verschiedenen Faktoren $a b$ [8] [9]:

- Qualität und stoffliche Eigenschaften,

- Einsatz- oder Verwendungsmöglichkeit,

- Verfügbarkeit ähnlicher oder kompetitiver Stoffe auf dem Markt und deren Kosten,

- Verfügbarkeit einer spezifischen Technologie oder Möglichkeiten für ihre Ent- wicklung, um einen Stoff der gewünschten Qualität zu erhalten,

- Beurteilung [technisch, ökologisch, wirtschaftlich] der Folgen einer Nicht-Rückgewinnung dieses Stoffes, und in diesem Fall,

- Beurteilung möglicher Langzeitrisiken und -haftung,

- logistische Überlegungen,

- arbeitshygienische Fragen, die mit der Rückgewinnung verbunden sind,

- Zeitabhängigkeit.

Die Randbedingungen für die Beurteilung von Recyclingmöglichkeiten mit Alternativen ändern sich ständig. Es ist daher angezeigt, diese regelmässig zu überprüfen.

Auf den ersten Blick scheint es zwischen Mengenbilanzen von üblichen chemischen Prozessen und Rückgewinnungs- bzw. Recycling-Prozessen keinen grundlegenden Unterschied zu geben. Wird jedoch die Mengenbilanz mit allen eingesetzten Ressourcen ergänzt, so fällt auf, dass der Rückgewinnungsprozess in der chemischen Industrie einerseits oft einen sehr hohen Energiebedarf hat und andererseits selber wieder zur neuen Abfallquelle wird. Dies ist verständlich, da es sich bei Rückgewinnungs- und Aufarbeitungsverfahren vornehmlich um Trenn- und Reinigungsoperationen handelt - und diese sind immer eine Energiesenke!

Es ist daher auch zu erwägen, welches der Nutzen einer Rückgewinnung für die Gesellschaft ist. Bei den derzeit tiefen Energiekosten wird der wahre Wert von nicht erneuerbaren und in beschränktem Masse vorhandenen Ressourcen nicht richtig gewichtet und verfälscht oft das Resultat zu ungunsten des Recycling. Die Frage der Rückführung eines Reaktionsrückstandes in den ursprünglichen Herstellprozess, oder dessen Wiedereinsatz in einem anderen Prozess, einem Prozessverbund oder bei Dritten, ändert nichts an der grundsätzlichen Zielsetzung der Ressourcenschonung. So kann unter Umständen die Nutzung des Wärmeinhaltes eines organischen Abfallproduktes anstelle dessen
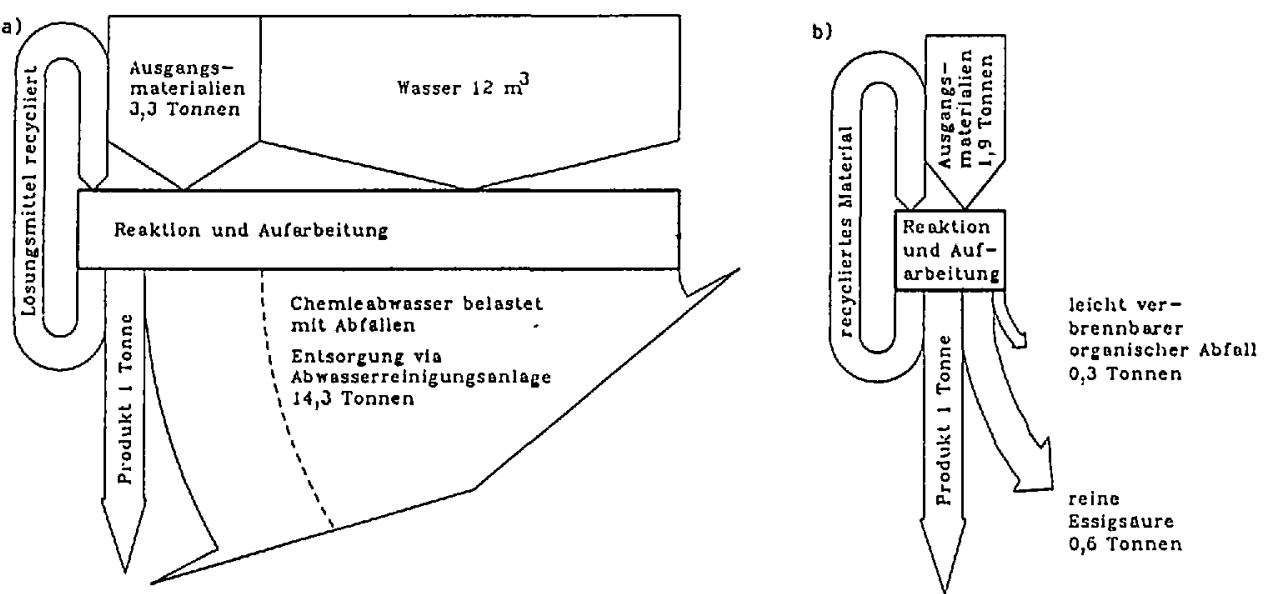

Fig. 3. a) Materialfluss vor der Verfahrensbearbeitung. b) Materialfluss nach der Verfahrensbearbeitung
Aufarbeitung gesamtökologisch die sinnvollere Lösung sein. Der Verband der Niederländischen Chemischen Industrie widmet diesem Thema seine besondere Aufmerksamkeit und schlägt ein systematisches und dynamisches Entscheidungsmodell vor, welches technische, ökologische und wirtschaftliche Gesichtspunkte berücksichtigt [10].

\section{Beispiele}

Im folgenden sind zur Erläuterung der o.e. Ausführungen drei Beispiele ausgewählt:

- Auf Ebene Prozeßstufe die Abfallminderung bei der Umwandlung einer organischen Säure in ein Amid; als Resultat der gezielten Verfahrensüberarbeitung einer Produktegruppe.

- Auf Ebene Produktegruppe das Resultat der Abfallminderung aus der bereits in den 70er Jahren gezielt durchgeführten Verfahrensüberarbeitung für die Herstellung von Buchstabensäuren. Dieses frühe Beispiel zeigt, dass auch bei sehr aufwendigem Mitteleinsatz eine beliebig grosse Abfallminderung nicht erreicht werden kann.

- Auf Ebene Produktionswerke das Resultat der Summe aller Verbesserungsmassnahmen auf die Abfallbilanz dieser Fabrik.

\subsection{Umwandlung einer organischen Säure} in ein Amid [11]

Das Beispiel erläutert die Anwendung der integrierten Verfahrensentwicklung auf eine einzelne Prozeßstufe. Eine gängige Methode für die Umwandlung einer organischen Säure in ein Amid führt über das entsprechende Säurechlorid. Bei der Herstellung eines Pharma-Zwischenproduktes wird das Säurechlorid durch Einwirken von Phosphortrichlorid auf die Säure ergebildete Säurechlorid sind sehr ätzend, weshalb bei der Synthese besondere Sicherhalten. Sowohl das Reagenz wie auch das 
heitsvorkehrungen erforderlich waren. Als unumgängliche Nebenprodukte entstanden organische Abfallstoffe, phosphorige Säure und grosse Mengen Salze. Die gesamte Abfallfracht wurde im Reaktionsabwasser via Abwasserreinigungsanlage entsorgt (vgl. Fig. 3).

In der Verfahrensforschung wurde das Verfahren überarbeitet und ein neuer Syntheseweg gefunden, der die kritische Stufe umgeht. Es entstand ein völlig neuer Prozess zur Herstellung des Amids, für den eine neue Anlage gebaut wurde. Diese ist so konzipiert, dass überhaupt kein Abwasser mehr anfällt. Die einzigen Stoffströme, welche die Anlage ausser dem gewünschten Amid verlassen, sind eine reine, andernorts einsetzbare Essigsäure sowie ein leicht verbrennbarer Destillationsrückstand, der zur Wärmenutzung beitragen wird. Eine derartige Überarbeitung einer Prozeßstufe erfordert den Einsatz von 1-2 Verfahrenschemikern während $c a$. $1 \mathrm{Jahr}$.

\subsection{Die Herstellung der Naphthalinsulfon- säurederivate (Buchstabensäuren)}

Ein gutes Beispiel für die Möglichkeiten, aber auch für die Grenzen der integrierten Verfahrensentwicklung ist die in der Fachliteratur bekannte [5] [12] Überarbeitung der z.T. vor etwa 80 Jahren eingeführten Produktionsverfahren für Buchstabensäuren. Diese Naphthalinsulfonsäurederivate sind wichtige Zwischenprodukte zur Synthese verschiedenster Farbstoffe.

Mitte der 70er Jahre wurden die traditionellen Produktionsverfahren überarbeitet im Hinblick auf den Bau des neuen Produktionskomplexes der Schelde-Chemie in
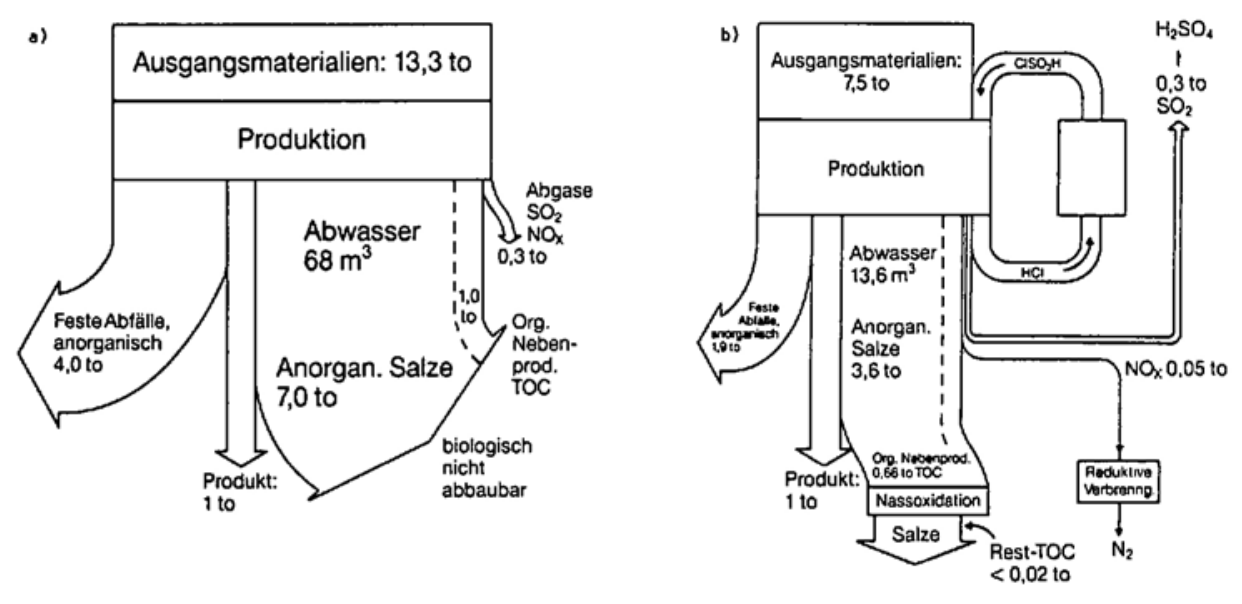

Fig. 4. Buchstabensäuren. a) Materialfluss der optimierten alten Verfahren. b) Materialfluss der neuen Verfahren

Norddeutschland (heute Bayer $A G$ ), welcher anfangs der 80er Jahre mit Erfolg in Betrieb genommen wurde. Die für die ganze Produktionsgruppe erfolgten Arbeiten hatten zum Ziel, durch Ausbeuteverbesserung, Einsatz neuester Technik, besseres Recycling und prozessintegrierte Abfallbehandlung die Gesamtabfallsituation zu verbessern. So wurde zum ersten Mal in der chemischen Industrie die völlig neu überarbeitete Technik der Nassoxidation für die Behandlung von prozeßspezifischen Mutterlaugen eingesetzt. Fig. 4 verdeutlicht das Resultat der Überarbeitung am Materialfluss der alten und der verbesserten Verfahren. Für die gesamte Produktegruppe ausgedrückt werden folgende Resultate erzielt:
20 Mio SFr.

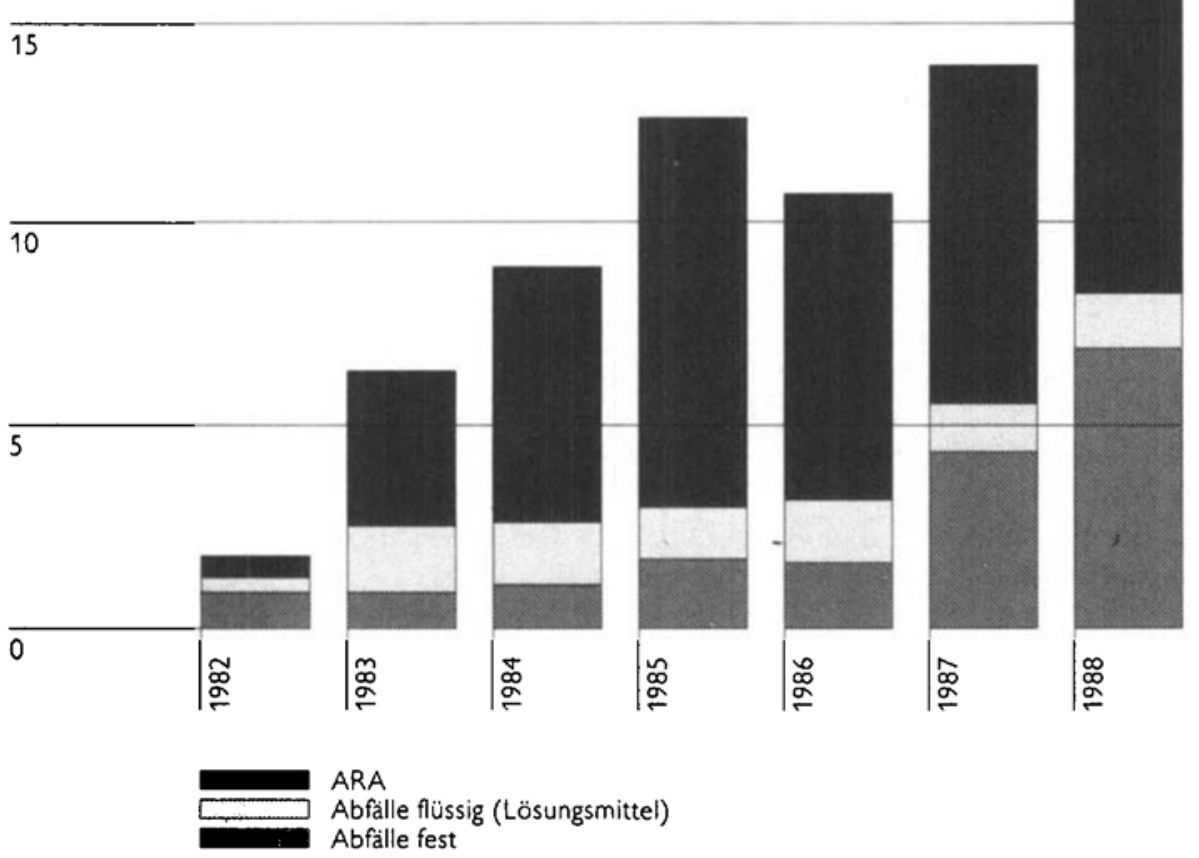

Fig. 5. Entsorgungskosten
- Minderung um 44\% des Rohstoffverbrauches von 13,3 $\mathrm{t}$ auf 7,5 $\mathrm{t}$ pro Tonne Fertigprodukt,

- Wiedereinsatz der Lösungsmittel im Prozess (d.h. keine Lösungsmittelverluste),

- Umwandlung von $\mathrm{HCl}$ (gasförmig) in Chlorsulfonsäure und Wiederverwendung im Prozess (d.h. keine HCl-Emissionen),

- Reinigung und Verflüssigung von $\mathrm{SO}_{2}$ Gas für externe Wiederverwendung (d.h. keine $\mathrm{SO}_{2}$-Emissionen),

- Reduktion der Gesamtabfallmenge um $50 \%$,

- Reduktion der organischen Last im Abwasser um $97 \%$ durch Verwendung der Nassoxidation,

- Vermeidung von organischen Abluftemissionen durch thermische Abfluftverbrennung.

Für das dargestellte Projekt waren während 3 Jahren $c a$. 20 Chemiker eingesetzt. Die Entwicklungskosten müssten heute mit über 80 Mio. SFr. veranschlagt werden.

3.3. Abfallbilanz eines Chemiewerkes [13] Im Werk Basel der Ciba-Geigy wurde versucht, die Ergebnisse aus der Summe der vorbeschriebenen Verbesserungsmassnahmen an der Abfallbilanz des Werkes zu messen. Die Untersuchungen erfolgten im Rahmen der Neukonzipierung der Abfallbewirtschaftung im Werk im Hinblick auf den geplanten neuen Sondermüllofen, aber auch angesichts der sehr stark steigenden Entsorgungskosten (Fig. 5). Diese sind für das gesamte Werk von 1982 bis 1988 von 2 auf $18 \mathrm{Mio}$. SFr. gestiegen (für Abwässer, feste Abfälle und Lösungsmittel). Die Kosten für die Entsorgung fester Abfälle stiegen im gleichen Zeitraum von 400 auf 2800 Franken pro Tonne. Diese Kosten werden nach dem Verursacherprinzip direkt dem Produkt angelastet. Daneben waren folgende Punkte für die erfolgreiche Abfallminderung von Bedeutung: 
- Einsatz nichtchlorierter alternativer Lösungsmittel anstelle von chlorierten Lösungsmitteln,

- Einsatz reinerer Rohstoffe,

- verbessertes Recycling, insbesondere bei Lösungsmitteln,

- verbesserte Prozessführung, teilweise durch Einsatz von moderner Stofftrennungsanalytik, die es erlaubt, Hauptund Nebenprodukte getrennt quantitativ zu erfassen,

- Einsatz neuer Technologien, z.B. der Umkehrosmose für die Behandlung von Mutterlaugen aus der Farbstoffproduktion,

- Weiterverwendung von Nebenprodukten und dadurch Entlastung der Abfallund Abwasserströme,

- Streichung von Produkten, deren Wirtschaftlichkeit aufgrund ihrer Umweltbelastung (Geruchsbelästigung, Entsorgung von Abluft, Abwasser und Abfällen, Arbeitshygiene) in Frage gestellt war,

- bessere Ausbildung und Motivation der Mitarbeiter

Als Resultat dieser Anstrengungen konnte die gesamte Abfallmenge pro Tonne Produkt in den Jahren 1982 bis 1988 um $40 \%$ gesenkt werden (Fig. 6). Die Menge von flüssigen und festen Abfällen pro Tonne Produkt konnte im gleichen Zeitraum ebenfalls um $40 \%$ verringert werden. Dabei tritt am deutlichsten die Reduktion der Salzlast bei der Farbstoffherstellung von nahezu $50 \%$ in den Vordergrund. Das Verhältnis der Abfallmengen (ohne Salze) zum Produktionsausstoss des Werkes Basel ist in absoluten Werten in Fig. 7 dargestellt.

\section{Konklusion und Ausblick}

Bei der Versorgung unserer Gesellschaft mit Dienstleistungen und Gütern jeder Art entstehen Abfälle. Diese entstehen auch beim Gebrauch vieler Güter sowie zu dem Zeitpunkt, wenn diese Güter ihrerseits ihre Qualität eingebüsst haben und nicht mehr verwendet werden können. Abfälle entstehen auch beim Betrieb vieler Umweltschutzanlagen. Diese verbrauchen zusätzlich Ressourcen und benötigen einen zum Teil erheblichen Einsatz an Energie. Es war seit jeher ein Anliegen der chemischen Industrie, die Abfallmenge aus der Produktion gering zu halten. Das Beispiel der Buchstabensäuren zeigt, dass bei sogenannten reifen, daher etablierten Verfahren auch mit erheblichem Entwicklungsaufwand die Abfallmenge bedeutend, aber nicht beliebig reduziert werden kann. Auch das Beispiel eines Produktionswerkes macht deutlich, dass bei Zusammenwirken aller abfallmindernden Massnahmen die spezifische Abfallmenge wohl reduziert, aber mit Entwicklung des Produktionsvolumens ebenfalls nicht beliebig tiefe Werte erzielt werden können. Bei ständig verbes-

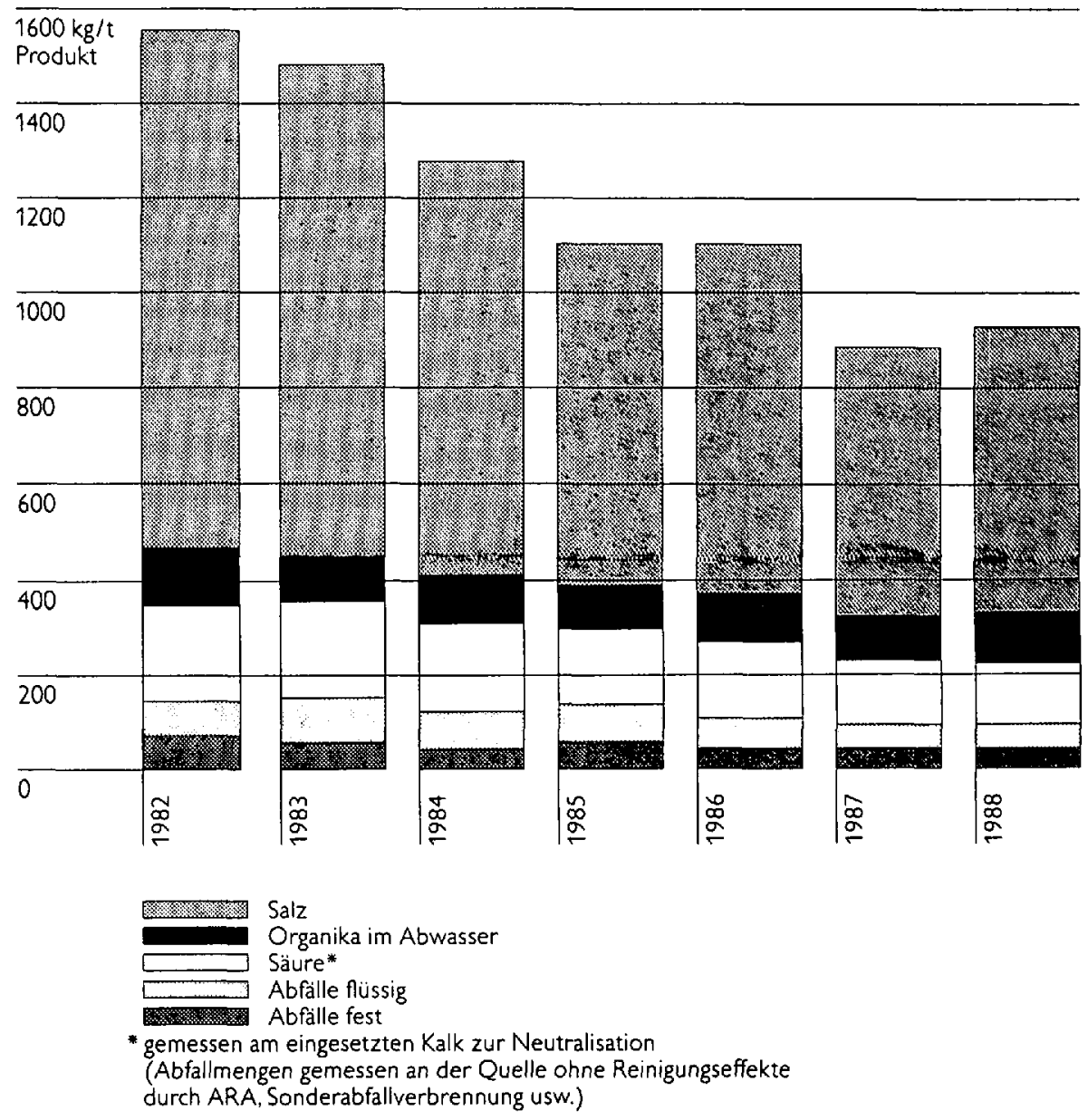

Fig. 6. Chemieabfälle Werk Basel

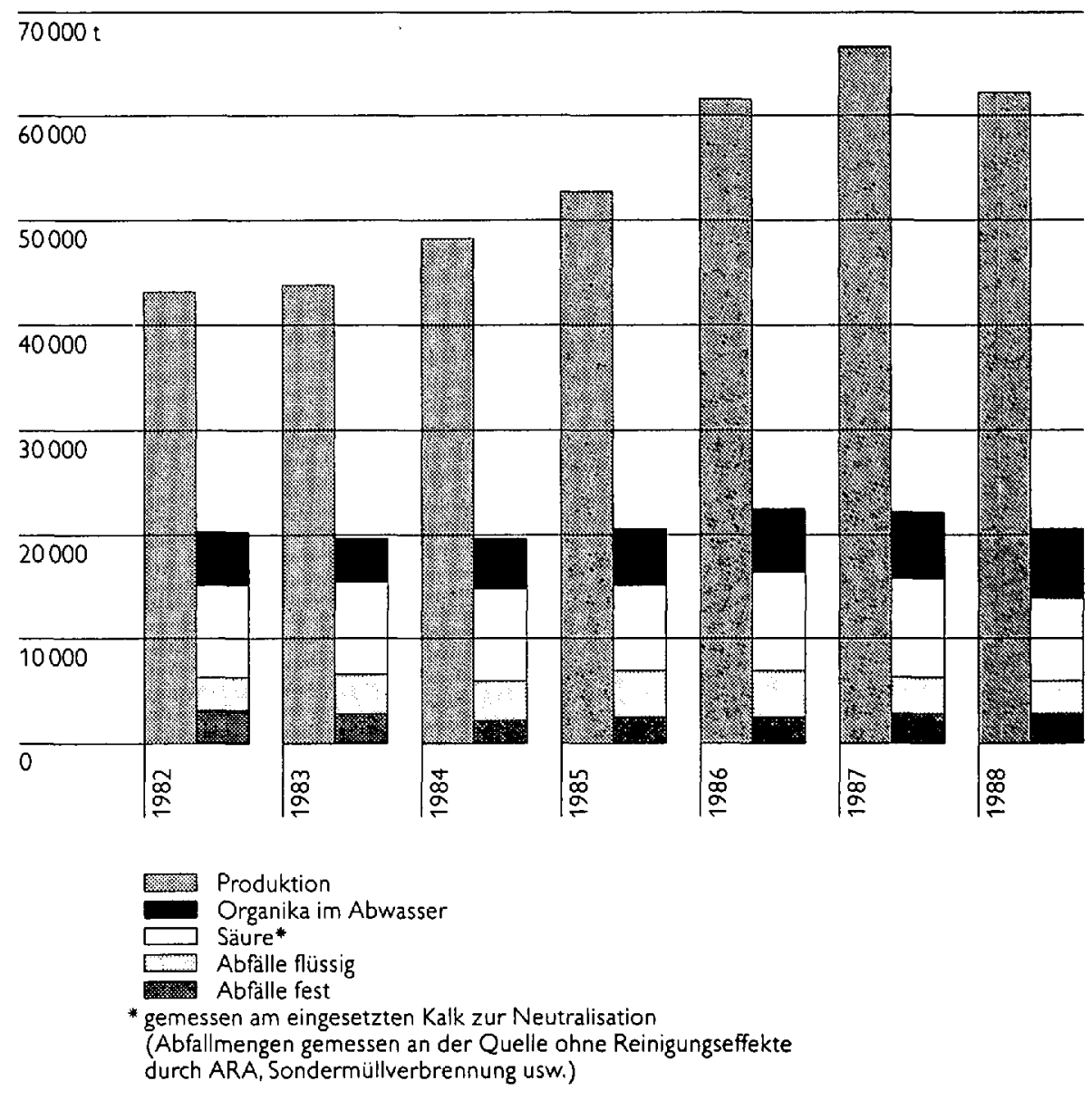

Fig. 7. Produktion und Abfälle (ohne Salz) absolut im Werk Basel 
serter Apparate-, Steuer- und Verfahrenstechnik jedoch ist der Optimierungsprozess zwischen Input und Output eines Herstellungsverfahrens nie abgeschlossen und das Potential an Effizienzverbesserung noch nicht ausgeschöpft.

Das Ziel einer im begrenzten System Erde immer noch exponentiell wachsenden Menschheit muss es sein, sich unter Schonung ihrer Ressourcen und im Einklang mit ihrer Umwelt zu entwickeln. Für die chemische Industrie stellt sich somit die Herausforderung, mit einem neuen Dienstleistungs- und Produkteangebot ihrer Aufgabe in der Gesellschaft gerecht zu werden. Dies bezieht sich auf den Nutzen der Produkte, Produktequalität, Herstellungsprozesse, Entsorgungstechnik, aber auch Logistik, Distribution und Marketing. Hiefür bedarf es ein sehr hohes Mass an Innovation und damit einen zunehmenden Bedarf an Forschung und Entwicklung. Dies wie- derum kann nur in einem gesunden wirtschaftlichen Klima gewährleistet werden. Zukunftsweisende Technologie muss zweckmässig, rationell und umweltfreundlich eingesetzt werden. Abfallminderung schliesslich ist integraler Bestandteil einer Strategie für ein qualitatives Wachstum und somit - für sich selber betrachtet schon eine Investition in die Zukunft.

[1] Leitbild für die Schweizerische Abfallwirtschaft, Bundesamt für Umwelt, Wald und Landschaft (BUWAL), Bern, Juni 1986; Technische Verordnung über Abfälle (TVA), Bern, Entwurf August 1988.

[2] Gesetz über die Vermeidung und Entsorgung von Abfällen (Abfallgesetz), Deutschland, 27. August 1986.
[3] G. Eigenmann, 'Gefährliche Sonderabfälle, Vergessen, Vernichten, Vermeiden', GDI-Tagung, Rüschlikon, 24.-25. November 1983.

[4] U. Gujer, Mitteilungen der Schweizerischen Gesellschaft für Boden- und Felsmechanik, 1986, 114.

[5] H. Bretscher, G. Eigenmann, E. Plattner, Chimia $1978,32(5)$

[6] U. Gujer, 'Gefahrenabwehrplan in einem Chemicwerk', IWS-Symposium über Umweltsicherheit beim Umgang mit wassergefährdenden Stoffen. Aachen, 3.-4. März 1988.

[7] G. Lipphardt, Chem.-lng.-Tech. 1989, 61 (11).

[8] G. Eigenmann, Wasser, Energie, Luft 75. Jahrgang $(7,8), 1983$

[9] U. Gujer, 'Das Leitbild für die schweizerische Abfallwirtschaft - Eine Herausforderung an die Wirtschaft', PRO AQUA PRO VITA, Fachtagung Basel, 6.-9. Juni 1989.

[10] Towards Integrated Recycling Management, Assoc. of the Dutch Chemical Industry (VNCl), November 1989.

[11] G. Acklin, R. Gygax, INFO CHEMIE, 1988, S.

[12] P. Caveng, 'Waste Minimization', IEB-Symposium, Genf, 20,--22. September 1989.

[13] J.R. Randegger, Ciba-Geigy-Zeitung Nr. 4, November 1989.
Chimia 44 (1990) 194-197

(C) Schweiz. Chemiker-Verband; ISSN 0009-4293

\section{Ist Entsorgung Aufgabe des Herstellers?}

\author{
Arthur Braunschweig*
}

Unsere westliche technische Gesellschaft hat einen beispiellosen Siegeszug über die ganze Welt erlebt - mit allen Vor- und Nachteilen, welche wir an dieser Stelle nicht diskutieren können. Ein untrügerisches Zeichen, dass dieser Siegeszug tatsächlich stattfand und weiter stattfindet, ist die Tatsache, dass Sie heute praktisch überall auf der Welt $A$ bfall finden können. Anfang dieses Jahrhunderts war Abfall in weiten Teilen der Welt noch ein unbekanntes Phänomen.

Auf diesem Hintergrund zur mir gestellten Frage: 'Ist Entsorgung Aufgabe des Herstellers?'. Nun: Faktisch ist heute die Entsorgung zumeist klar nicht Aufgabe des Herstellers. Mit Recht können Sie nun einwenden, gerade in der chemischen Industrie gebe es massenhaft Beispiele, wo der Hersteller sehr wohl Hinweise auf die weitere Verwendung eines Stoffes mache. So müssen doch z.B. die Hersteller von Medikamenten oder Pflanzenbehandlungsmitteln deren weiteres Verhalten im menschlichen Körper resp. in Pflanzen und im

\footnotetext{
*Korrespondenz: Dr. A. Braunschweig

Ö.B.U.

Seitzstr. 13

CH 9000 St. Gallen
}

Ackerfeld berücksichtigen; und dies ist ja eigentlich gerade die Entsorgung dieser Produkte.

Doch wie verhält es sich z.B. bei diesem flüssigen Rachendesinfektans? Der Patient gurgelt damit. Und wohin gelangt es anschliessend? Auf der Packung steht 'Nicht schlucken'. Also wird es wohl in den Abguss gespuckt und gelangt in die Kanalisation. Und hier wäre ich - vorsichtig gesagt - erstaunt, wenn die langfristige Wirkung der Entsorgungsproblematik befriedigend im Griff wäre. Vielleicht könnte das Mittel auch mit einem Zerstäuber appliziert werden, was sparsamere Dosierung erlauben und Entsorgungsprobleme nach Gebrauch vermeiden würde: Das Mittel würde dann nämlich einzig am gewünschten Ort appliziert. (Den Fall ungebrauchter Medikamente wollen wir später kurz erwähnen.) Auch wenn ich mich im Fall dieses Produktes täuschen sollte - was mich freuen würde - meine ich doch, dass heute Entsorgung meist vom Hersteller nicht genügend betrachtet wird.

Einige Bemerkungen zu den Rahmenbedingungen

- Die folgenden Überlegungen sind im weiteren Sinne Teil einer 'Förderung des

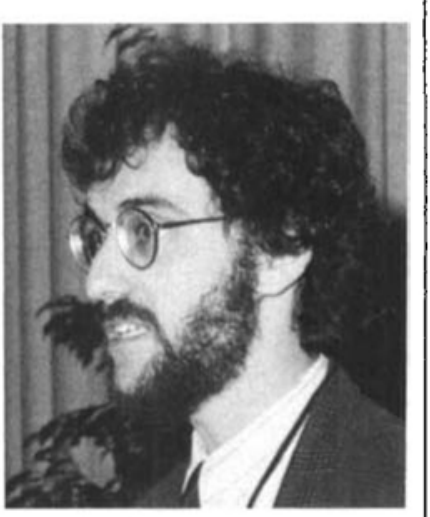

Arthur Braunschweig: Geboren 1959. Nach Wirtschaftsgymnasium und Auslandaufenthalt Studium und Doktorat an der Hochschule St. Gallen, letzteres zum Thema 'Ökologische Buchhaltung', einer Methodik der ökologischen Bilanzierung. 3 Jahre bei $I B M$. Heute Lehrbeauftragter und Projektmitarbeiter an der HSG sowie hauptamtlich Geschäftsführer der Ö.B.U., welche u.a. durch unternehmerische Aktionsgruppen und Informationsvermittlung in den Mitgliedunternehmen ökologisch sinnvolle Veränderungen unterstützen und anstossen will.

qualitativen Wachstums' oder einer Strategie hin zu einer 'ökologischen Marktwirtschaft'.

- Das Verhalten der Unternehmungen ist zu grossen Teilen auch abhängig von den herrschenden Rahmenbedingungen. Auf diese werde ich im folgenden aus zeitlichen Gründen nicht eingehen können, obwohl sie von entscheidender Bedeutung sind.

- Es versteht sich letztlich von selbst, dass ich Entsorgung als Teil der gesamten globalen Umweltpolitik verstehe, ohne aber auf die anderen Facetten der Umweltprobleme eingehen zu können. 